[CONTRIBUtion FROM THE ChEMICAL LABORATORY OF THE UNIVERSitY OF Michigan.]

\title{
BENZYL ETHERS OF CARBOHYDRATES.
}

\author{
By M. Gomberg and C. C. BuchleER.
}

Received May 16, 1921.

In a previous communication ${ }^{1}$ it was shown that benzyl esters could be prepared in good yields, and a pure condition, from benzyl chloride and the sodium salt of the corresponding acid dissolved in water; in aqueous solution, also, benzyl ethers, amines, benzonitriles, etc., were prepared. In testing further the applicability of this method of procedure, we have now studied the action of benzyl chloride upon carbohydrates.

Alkylation of carbohydrates had been limited for a time, since 1893, to $\mathrm{E}$. Fischer's ${ }^{2}$ method of making alkyl glucosides by the condensation of the carbohydrate and the alcohol through the agency of hydrochloric acid. Under these circumstances, the alkyl group becomes attached to the oxygen in the aldehydic or ketonic group, and can be readily separated by hydrolysis. Later, Maquenne ${ }^{3}$ prepared $\beta$-methylglucoside by the action of methyl sulfate and sodium hydroxide on glucose. Purdie and Irvine ${ }^{4}$ then showed that the hydrogen atoms in the four alcoholic hydroxyl groups also could be replaced by alkyl groups by the use of silver oxide and alkyl iodide. As was to be expected, the resulting ethers are not subject to the influence of hydrolyzing agents under ordinary conditions, and consequently they exceed greatly in stability the esters of the carbohydrates. Irvine and his co-workers have successfully methylated in this way a number of carbohydrates, partial alkylation occurring readily and complete alkylation being achieved by repeated treatment in several stages. The use of methyl sulfate for making methyl ethers of cellulose was described by Denham and Woodhouse in $1913 . .^{5}$ In the same and in the following year appeared brief abstracts of patented processes ${ }^{6}$ for methyl and ethyl cellulose ethers, using alkyl sulfates or halides. More recently Haworth ${ }^{7}$ showed that not only cellulose, but various sugars can be alkylated through the agency of methyl sulfate and aqueous sodium hydroxide instead of the expensive materials as in the method of Purdie and Irvine. Since then, the methyl sulfate procedure alone, or that and followed by the more drastic treatment with methyl iodide and silver oxide, has been used by

${ }^{1}$ Gomberg and Buchler, This Journai, 42, 2059 (1920).

${ }^{2}$ Fischer, Ber., 26, 2400 (1893).

${ }^{3}$ Maquenne, Bull. soc. chim., 33, 469 (1905).

${ }^{4}$ Purdie and Irvine, J. Chem. Soc., 83, 1021 (1903).

${ }^{5}$ Denham and Woodhouse, ibid., 103, 1735 (1913); 105, 2357 (1914).

${ }^{6}$ Lilienfeld, J. Chem. Ind., 32, 420 (1913); Fr. pat. 447,974, C. A., 7, 3839 (1913);

Erit. pat. 12,854. Dreyfus, J."Soc. Chem. Ind., 33, 248 (1914); Fr. pat. 462,274.

"Haworth, J. Chem. Soc, 107, 8 (1915). 
Denham and Woodhouse ${ }^{8}$ on cellulose, by Haworth ${ }^{9}$ on maltose and on cellobiose, by Irvine ${ }^{10}$ on inulin, and by Karrer ${ }^{11}$ on starch.

In addition there are several more recent patents. ${ }^{12}$ All these patents make, of course, very broad claims for alkylating agents in general, but mention of specific examples is limited, so far as we are aware, to examples of methyl and ethyl ethers only, from cellulose and from starch. Benzyl ethers specifically we find mentioned only in Brit. pat. 149,320, "for carbohydrates of the general formula $\mathrm{C}_{6} \mathrm{H}_{10} \mathrm{O}_{5}$;" the abstract of this patent came into our hands after all the work described in this paper had been completed. Here, again, the working example described in this last patent is that of ethyl ethers.

Benzyl chloride; we find, lends itself exceedingly well to the alkylation of all classes of carbohydrates. No attempt was made for the present to ascertain the best conditions for the most complete alkylation, but merely to determine how general this reaction is. Methylglucoside, sucrose, lactose, inulin, dextrin, starch and cellulose all form benzyl ethers more or less readily. The general procedure was to dissolve, or suspend, the individual carbohydrate in water which contained enough sodium hydroxide to keep the solution alkaline throughout the whole period of reaction; benzyl chloride was added in the proportion of slightly more than $1 \mathrm{~mol}$ of the chloride for each hydroxyl group in the carbohydrate used, and the mixture well stirred. After the reaction was deemed to have been completed, the excess of benzyl chloride was removed by steam distillation, and the subsequent treatment was as described below, for the individual carbohydrates. For analysis, the purified products were dried at $100^{\circ}$ for 6 to 10 hours.

\section{Experimental.}

Benzylation of Glucose.-Several preliminary experiments with glucose gave unsatisfactory results, due no doubt to the detrimental action of the alkali upon the glucose. $\alpha$-Methylglucoside was then prepared by the method of E. Fischer, except that we used much less methyl alcohol and the latter contained only $10 \%$ of hydrogen chloride instead of being saturated with it. The glucose solution was allowed to stand until it ceased to reduce Fehling's solution, and a $50 \%$ yield of $\alpha$-methylglucoside was obtained.

When the glucoside was benzylated at $85^{\circ}$ to $90^{\circ}$, an oil was obtained which was insoluble in water but soluble in benzene. The results of the

8 Denham and Woodhouse, J. Chem. Soc., 119, 77 (1921).

${ }^{9}$ Haworth, ibid., 115, 809 (1919); 119, 193 (1921).

${ }^{10}$ Irvine, ibid., 117, 1474 (1920).

"Karrer, Helvetica chim. acta, 3, 620 (1920).

12 Lilienfeld, $C . A, 10,2145$ (1916); U. S. pat. 1,188,376. C. A., 14, 3251 (1920); U. S. pat. 1,350,820. C. A., 15, 436 (1921); Brit. pat. 149,320 . 
elementary analysis indicate that this must have been a mixture consisting largely of dibenzyl-methylglucoside with some higher benzylated products. The following procedure was employed in the separation of the reaction mixture into its components.

$9.7 \mathrm{~g}$. (1 mol) of methylglucoside, $12 \mathrm{~g}$. (6 mols) of sodium hydroxide in $100 \mathrm{cc}$. of water, and $27.5 \mathrm{~g}$. ( 4.4 mols) of benzyl chloride were heated with stirring at $90^{\circ}$ to $95^{\circ}$ for 6 hours. The excess of benzyl chloride was removed by steam distillation, the aqueous solution was decanted from the oily reaction product and worked up separately. The oil was then stirred up with 4 successive portions of $100 \mathrm{cc}$. each of hot water, in order to dissolve out any benzyl alcohol that might have been present. The residue was taken up in benzene, the solution dried with calcium chloride and the solvent completely removed. The oily product was then extracted several times with petroleum ether, b. p. $50^{\circ}$ to $70^{\circ}$, which dissolved out about half of the material. On evaporation of the petroleum ether $1.5 \mathrm{~g}$. of residue of a mobile consistency was obtained, which proved to be the completely benzylated product, tetrabenzyl-benzylglucoside. (II). The portion which did not dissolve in petroleum ether, also about $1.5 \mathrm{~g}$., became a stiff paste, as it cooled. It proved to be dibenzyl-methylglucoside. (I).

Analyses I. Calc. for $\mathrm{C}_{7} \mathrm{H}_{12} \mathrm{O}_{6}\left(\mathrm{C}_{7} \mathrm{H}_{7}\right)_{2}: \mathrm{C}, 67.37 ; \mathrm{H}, 6.95$. Found: $\mathrm{C}, 67.07$; $\mathrm{H}, 6.81$.

$\mathrm{H}, 6.78$.

II. Calc. for $\mathrm{C}_{6} \mathrm{H}_{7} \mathrm{O}_{5}\left(\mathrm{C}_{7} \mathrm{H}_{7}\right)_{5}: \mathrm{C}, 78.10 ; \mathrm{H}, 6.65$. Found: $\mathrm{C}, 78.25$;

The aqueous solution decanted from the mixture of the insoluble benzylated giucosides was examined for the presence of water-soluble products, and was found to contain $2.5 \mathrm{~g}$. of unchanged methylglucoside, and $2 \mathrm{~g}$. of benzylglucoside. The aqueous solution, after neutralization, was evaporated to dryness, extracted with $95 \%$ alcohol, the filtered alcoholic solution evaporated to dryness and again extracted with absolute alcohol; the latter was evaporated to dryness. The residual mixture was washed with benzene in order to remove any benzyl alcohol, and the two glucosides were separated from each other by means of acetone, which dissolved only the benzylglucoside and left behind a white crystalline mass, readily identified as the methylglucoside by its melting point, solubilities and composition. The product soluble in acetone was analyzed with the following result.

Analysis. Calc. for $\mathrm{C}_{6} \mathrm{H}_{11} \mathrm{O}_{6}\left(\mathrm{C}_{7} \mathrm{H}_{7}\right): \mathrm{C}, 57.78 ; \mathrm{H}, 6.67$. Found: $\mathrm{C}, 56.71 ; \mathrm{H}, 6.89$.

Thus, under the conditions described, the action of benzyl chloride upon methylglucoside yielded three products: benzylglucoside, dibenzylmethylglucoside and tetrabenzyl-benzylglucoside.

The benzylglucoside showed the properties described for this compound by $\mathrm{E}$. Fischer ${ }^{13}$ who prepared it from benzyl alcohol and glucose. It was characterized by a bitter taste and was found to be soluble in water, alcohol, benzene, chloroform, ethyl acetate and acetone; insoluble in ether and in petroleum ether. It did not reduce Fehling's solution, but was readily hydrolyzed by $8 \%$ hydrochloric acid upon boiling the solution, and gave rise to a strongly reducing carbohydrate; it was soluble in conc. hydrochloric acid and was hydrolyzed by it in the cold.

The substance of the composition of dibenzyl-methylglucoside was a semi-solid material, soluble in benzene and chloroform; it gave a cloudy

${ }^{13}$ Fischer, Ber., 26, 2410 (1893). 
solution with acetone and with ethyl acetate, was only slightly soluble in alcohol and in acetic acid, and insoluble in absolute ether and in petroleum ether. It was found to be without effect upon Fehling's solution; upon boiling with $8 \%$ hydrochloric acid for 1 hour it yielded a product still only little soluble in water, but strongly reducing.

Tetrabenzyl-benzylglucoside is the completely benzylated product. Assuming the usually accepted butylene oxide formula for the glucosides, the constitution of the benzylated product is,

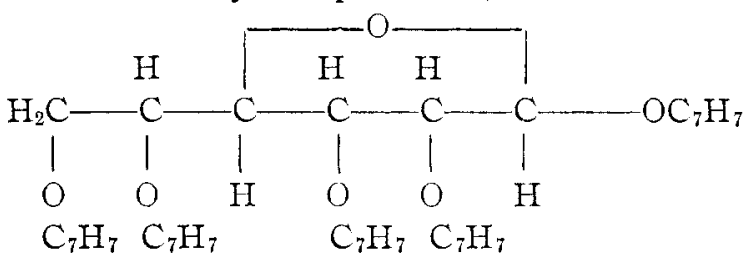

This substance is an oil, as is the completely methylated $\alpha$-methyl-glucoside, ${ }^{14}$ and is soluble in a greater variety of organic solvents than the partially benzylated compound, being soluble even in ether and in petroleum ether. It is not reducing, but becomes so upon boiling for an hour with dil. hydrochloric acid. In cold conc. hydrochloric acid it remains largely undissolved, and only after two days' standing does the solution show appreciable action upon Fehling's reagent.

Benzylation of Sucrose.-It was found difficult to judge the progress of the reaction in the case of sucrose, since the resulting products remain dissolved in the excess of the benzyl chloride.

One tnol of sucrose, $8.8 \mathrm{mols}$ of benzy 1 chloricle and $12 \mathrm{mols}$ of sodium hydroxide $(10 \%)$ were heated for 4.5 hours at $85^{\circ}$. After steam distillation the residual gum was dissolved in a mixture of chloroform and benzene, the solution dried, and absolute ether added to the concentrated solution. The precipitated granular powder was rubbed up with fresh quantities of absolnte ether, filtered, washed and dried. Analysis showed that the substance was a mixture of the monobenzyl derivative with higher alkylated products. In the next run, with materials in the same proportion as above, the heating was done at $100^{\circ}$ for 3 hours and the reaction mixture was worked up in the same manner. The product consisted almost wholly of dibenzyl sucrose, containing $58.2 \%$ of carbon, and $6.1 \%$ of hydrogen. In the next experiment the action was more drastic. The mixture was heated for 4 hours at $100^{\circ}$, the aqueous layer was siphoned off and to the oil fresh benzyl chloride and sodium hydroxide solution were added. After further heating for 4 hours the mixture was worked up as described. There were no watersoluble benzyl sucroses formed; those insoluble in water were dissolved in chloroform. After evaporating all the chloroform the crude gummy product was resolved, on treattnent with absolute ether, into two components: an insoluble white granular solid which proved to be dibenzyl sucrose, and a portion soluble in ether, an oil, which analyzed for a pentabenzyl sucrose. This oil, before analysis, was extracted several times with petrolaum ether in order to remove any benzyl alcohol that might, perhaps, be present.

Analyses. Solid. Calc. for $\mathrm{C}_{12} \mathrm{H}_{20} \mathrm{O}_{11}\left(\mathrm{C}_{7} \mathrm{H}_{7}\right)_{2} ; \mathrm{C}, 59.77 ; \mathrm{H}, 6.51$. Found: $\mathrm{C}$, $59.73 ; \mathrm{H}, 6.14$.

I,iquid. Calc. for $\mathrm{C}_{12} \mathrm{H}_{17} \mathrm{O}_{11}\left(\mathrm{C}_{7} \mathrm{H}_{7}\right)_{5}: \mathrm{C}, 71.21 ; \mathrm{H}, 6.56$. Found: $\mathrm{C}, 70,09 ; \mathrm{H}, 6.53$.

${ }^{14}$ Purdie and Irvine, J. Chem. Soc., 83, 1030 (1903). 
The dibenzyl and the pentabenzyl sucrose possess a most persistent bitter taste. Although they are practically insoluble in water, yet the supernatant aqueous layer acquires in a short time the intensely bitter taste. Neither of these compounds reduces Fehling's solution. On boiling with $8 \%$ hydrochloric acid small amounts of the substances are hydrolyzed and the solutions acquire reducing properties. In cold conc. hydrochloric acid the benzyl compounds dissolve partially. The solutions become dark colored and after 2 to 3 hours' standing show reducing properties, the dibenzyl derivative apparently hydrolyzing more readily than the pentabenzyl. The two compounds were found to be soluble in alcohol, acetic acid, chloroform, chlorohydrin, ethyl acetate, benzene, and nitrobenzene. The pentabenzyl was soluble also in ether and in acetone, while the dibenzyl sucrose was only slightly soluble in acetone and not at all in ether. Both were found to be insoluble in petroleum ether.

The benzyl sucroses furnish excellent examples of the influence of the benzyl group upon the physiological properties of substances. It is generally recognized that, in a substance possessing sweet taste, the entrance of a phenyl ${ }^{15}$ or benzyl group into the molecule tends to decrease the sweetness, and it often inverts the taste completely from the sweet to the bitter. Methyl- and ethylglucosides, $\alpha$ - and $\beta$-modifications, as well as the similar glucosides of other monoses, are all sweet; but with increase in weight of the alcohol molecule the taste begins to change, and the glucosides of benzyl alcohol, benzaldehyde, salicyl alcohol, of phenolssynthetic and natural glucosidic products-are in the great majority of instances bitter. Now, in the benzyl sucroses here described, the benzyl group can not be linked to an aldehydic or ketonic oxygen, since none such is present in sucrose, and yet these benzyl ethers compare well with quinine or picric acid in taste.

Benzyl Dextrin.-The dextrin used was a product known to have come from pure potato starch and was completely soluble in water.

One mol of dextrin, 3.5 mols of benzyl chloride and 4.5 mols of sodium hydroxide $(9 \%)$, were heated at $80^{\circ}$ to $85^{\circ}$ for 4 hours, and treated in the usual manner. Upon addition of ether to the concentrated chloroform solution of the reaction mixture, from $16 \mathrm{~g}$. of dextrin $18 \mathrm{gr}$. of the insoluble benzyl derivative was obtained. Elementary analysis gave results for carbon only slightly in excess of that required for a substance of the formula $\mathrm{C}_{12} \mathrm{H}_{19} \mathrm{O}_{10} \mathrm{C}_{7} \mathrm{H}_{7}$. The benzyl compound obtained was a white amorphous tasteless powder, melting at about $208-10^{\circ}$. It was insoluble in water, acetone, ether; slightly soluble in benzene, and nitrobenzene. It swelled up in alcohol and ethyl acetate and with chloroform, chlorohydrin, and acetic acid gave colloidal transparent solutions which passed through filter paper with difficulty. It did not reduce Fehling's solution, was slowly hydrolyzed by dil. hot hydrochloric acid, or when in contact with the cold concentrated acid.

${ }^{15}$ G. Cohn, "Organische Geschmackstoffe," Franz Siemenroth, 1914, pp. 102, 243-266. 
Benzylation of Starch.-The experiments were performed on two samples of starch of known origin, potato and corn starch. In each case the reaction temperature was $85^{\circ}$ to $90^{\circ}$, the relative proportions of the reacting ingredients were the same as with dextrin, and yet the resulting products were not alike: potato starch took up one benzyl group for each $\mathrm{C}_{12}$ molecule, while corn starch took up two benzyl groups.

The crude gummy reaction product from potato starch, after thorough air drying, was dissolved in chlorohydrin, and the solution was diluted with chloroform and filtered through paper in a jacketed funnel. The benzyl compound was precipitated from its solution by the addition of ether, and the white powder was rubbed up in a mortar with several portions of fresh ether, filtered and dried. The yield was $8 \mathrm{~g}$. of the powder from $8 \mathrm{~g}$. of starch. The corn starch gave a crude product similar in appearance to that obtained from the potato starch. It was divided into portions: one portion was purified by solution in chlorohydrin and precipitation therefrom by meats of ether, the second portion by solution in acetic acid and reprecipitation - ether. The yield was $20 \mathrm{~g}$. of the benzyl compound from $16 \mathrm{~g}$. of the corn starch.

Analyses. Potato starch. Calc. for $\mathrm{C}_{12} \mathrm{H}_{19} \mathrm{O}_{10}\left(\mathrm{C}_{7} \mathrm{H}_{7}\right): \mathrm{C}, 55.07 ; 1.6 .28$. Found: C, $55.85 ; \mathrm{H}, 6.12$.

Corn starch (chlorohydrin). Calc. for $\mathrm{C}_{12} \mathrm{H}_{18} \mathrm{O}_{10}\left(\mathrm{C}_{7} \mathrm{H}_{7}\right)_{2}: \mathrm{C}, 61.2 \mathrm{H}, 6.35$. Found: C, 61.38; H, 6.38.

Corn starch (acetic acid). Calc. for $\mathrm{C}_{12} \mathrm{H}_{18} \mathrm{O}_{10}\left(\mathrm{C}_{7} \mathrm{H}_{7}\right)_{2}: \mathrm{C}, 61.92 ; \mathrm{H}, 6.35$. und: C, $60.43 ; \mathrm{H}, 6.31$.

'The monobenzyl starch melted at $200^{\circ}$ to $203^{\circ}$, the dibenzyl at $203^{\circ}$ to $205^{\circ}$. Bor. were soluble in chlorohydrin and acetic acid, and insoluble in alcohol, acetone, ether, chloroform, benzene, nitrobenzene, ethyl acetate and benzyl benzoate. Filter paper, dipped into a sulution of the benzyl starch becomes tough and water-proof when dry. With Fehling's solution the benzyl starches behave like benzyl dextrin.

Inulin, also, was benzylated in like manner as the starches, and the resulting product analyzed a little short for a substance of the formula $\mathrm{C}_{12} \mathrm{H}_{19} \mathrm{O}_{10}\left(\mathrm{C}_{7} \mathrm{H}_{7}\right)$.

Benzylation of Cellulose.-Cellulose, unless previously subjected to one of the several so called "hydration" processes, was found to suffer but slight benzylation under the conditions as described for the other carbohydrates. It was found that benzylated cellulose was insoluble in Schweitzer's reagent, and this fact greatly facilitated the purification of the alkylated products.

(1) Absorbent cotton, subjected to previous hydration by means of zinc chloride and hydrochloric acid and, of course, carefully freed from these, was benzylated in the usual manner at $90^{\circ}$ to $95^{\circ}$. The resulting product was still partially soluble in cuprammonium solution, and the elementary analysis indicated an extent of benzylation less than one benzyl group for each $\mathrm{C}_{12}$ molecule.

(2) Five g. of absorbent cotton was dissolved in Schweitzer's reagent and the solution allowed to stand overnight. The cellulose was precipitated by the addition of alcohol, and was thoroughly freed from copper. It was then digested for 12 hours with a solution of sodium hydroxide (11 g. of alkali in $73 \mathrm{cc}$. of water); $22 \mathrm{~g}$. of benzyl chloride was added and the mixture was heated for 7 hours at $90^{\circ}$ to $95^{\circ}$. After steam distillation, the product was thoroughly washed with water, alcohol and ether. The gray powder was then insoluble in cuprammonium solution, in chloroform, chlorohydrin, acetone, acetic acid, benzene and nitrobenzene. 
Analysis. Calc. for $\mathrm{C}_{12} \mathrm{H}_{19} \mathrm{O}_{10}\left(\mathrm{C}_{7} \mathrm{H}_{7}\right): \mathrm{C}, 55.07 ; \mathrm{H}, 6.28$. Found: C, 54.34 ; H, 6.24 .

(3) Five g. of absorbent cotton was converted into hydrocellulose by means of hydrogen chloride. ${ }^{16}$ After completely washing out the acid from it, the material was digested for 3 hours with $50 \mathrm{cc}$. of $15 \%$ sodium hydroxide solution; then $10 \mathrm{cc}$. of water and $18 \mathrm{~g}$. of benzyl chloride were added and the mixture was heated for 4 hours. The benzylated product was digested for several hours with cuprammonium solution, filtered, washed, digested in several changes of dil. hydrochloric acid, and washed with water, alcohol and ether. The benzylated product, amounting to $4 \mathrm{~g}$., consisted of white very short fibres. Hydrocellulose is generally assumed to be a further degradation product of cellulose than the "cellulose hydrates." The composition of hydrocellulose is given as $n\left(\mathrm{C}_{6} \mathrm{H}_{10} \mathrm{O}_{5}\right)+\mathrm{H}_{2} \mathrm{O}, n$ varying from 2 to $6,{ }^{17}$ and this relative composition is believed to be retained in the various derivatives of hydrocellulose. ${ }^{18}$ For our purposes we may assume $n$ equal to 4 and shall not be far from the truth.

Analysis. Calc. for $\left.2 \mathrm{C}_{12} \mathrm{H}_{19} \mathrm{O}_{10} \mathrm{C}_{7} \mathrm{H}_{7}\right) \cdot \mathrm{H}_{2} \mathrm{O}: \mathrm{C}, 53.90 ; \mathrm{H}, 6.38$. Found: C, 53.60; $\mathrm{H}, 6.58$.

(4) Absorbent cotton was soaked in a $15 \%$ sodium hydroxide solution for 3 days, and then squeezed until the cotton retained about 3 times its weight of the alkaline solution. The alkali cellulose was heated for 5 hours at $100^{\circ}$ with an excess of benzyl chloride. After steam distillation and preliminary purification, the product was digested for 2 days with cuprammonium solution and then purified as described above. Throughout the whole treatment the fibrous structure of the cotton was retained.

Analysis. Calc. for $\mathrm{C}_{12} \mathrm{H}_{19} \mathrm{O}_{10}\left(\mathrm{C}_{7} \mathrm{H}_{7}\right): \mathrm{C}, 55.07 ; \mathrm{H}, 6.28$. Found: C, 57.97; $\mathrm{H}, 6.37$.

(5) More homogeneous products were obtained with filter-paper cellulose. Four g. of pure ashless paper, cut into small pieces, was soaked for 12 hours in $30 \mathrm{cc}$. of $20 \%$ sodium hydroxide solution; $45 \mathrm{cc}$. of water was then added and the mixture was heated for an hour at $90^{\circ}$ to $95^{\circ}$ with no apparent change. Seventeen $g$. of benzyl chloride was introduced and the heating continued. After 4 hours the paper began to change visibly, gathering into a clump. After another hour of heating, the mixture was worked up in the usual way, digested for a day in Schweitzer's reagent and purified.

Analysis. Calc. for $\mathrm{C}_{12} \mathrm{H}_{18} \mathrm{O}_{10}\left(\mathrm{C}_{7} \mathrm{H}_{7}\right): \mathrm{C}, 55.07 ; \mathrm{H}, 6.28$. Found: C, 55.38; $\mathrm{H}, 6.45$.

(6) Filter-paper cellulose was subjected to a more energetic, two-stage, benzylation. Five g. of the paper was digested for 2 days at room temperature in a solution of $10 \mathrm{~g}$. of sodium hydroxide in $30 \mathrm{cc}$. of water, $30 \mathrm{~g}$. of benzyl chloride was added and the mixture was heated at $100^{\circ}$ for 7 hours. After distillation with steam and thorough washing with water, alcohol and ether, the dried material weighed $6 \mathrm{~g}$. A portion of this was saved for purposes of analysis, and the remaining $4.5 \mathrm{~g}$. was subjected once more to the same treatment with alkali and benzyl chloride. It yielded, after washing with alcohol and ether, $5 \mathrm{~g}$. of dry product which however proved to be not entirely homogeneous. On digestion with ethyl acetate most of it, but not all, went into solution and was reprecipitated from the clear filtrate, upon the addition of absolute alcohol, as a white, horny substance. The one-stage benzylated product was treated, before analysis, with cuprammonium solution.

Analyses. One-stage benzylation. Calculated for $\mathrm{C}_{12} \mathrm{H}_{17} \mathrm{O}_{10}\left(\mathrm{C}_{7} \mathrm{H}_{7}\right)_{3}: \mathrm{C}, 66.67$; $\mathrm{H}, 6$.39. Found: C, 66.10; H, 6.41.

${ }^{16}$ Cross and Bevan, "Cellulose," Longmans Green and Co., 1918, pp. 54, 254.

${ }_{17}$ Meyer u. Jacobson, "Organische Chemie," Veit and Co., 1913, I, 2, p. 1044.

${ }^{18}$ Worden, "Technology of Cellulose Esters," Van Nostrand Co., 1916, VIII, p. 2541. 
Two-stage benzylation. Calculated for $\mathrm{C}_{12} \mathrm{H}_{16} \mathrm{O}_{10}\left(\mathrm{C}_{7} \mathrm{H}_{7}\right)_{4}: \mathrm{C}, 70.17 ; \mathrm{H}, 6.58$. Found: $\mathrm{C}, 69.74 ; \mathrm{H}, 6.32$.

It does not appear probable that the product showing the composition of tribenzyl cellulose is a mixture of di- and tetrabenzyl derivatives. The mono benzyl compound did not show signs of softening when heated to $250^{\circ}$, the tribenzyl substance melted at $208^{\circ}$ to $10^{\circ}$, while the tetra benzyl compound softened at $165^{\circ}$, and melted at $175^{\circ}$ to $77^{\circ}$. The tribenzyl substance appeared as fully insoluble in the various organic solvents as the monobenzyl derivative, while the tetrabenzyl cellulose proved soluble in chlorohydrin, chloroform, nitrobenzene, ethyl acetate, and was gelatinized by acetone.

The three benzyl derivatives do not reduce Fehling's solution. On treating with dilute acid, the monobenzyl and the tribenzyl compounds yield after 1 hour soluble reducing carbohydrates, while tetrabenzyl cellulose does not do so even after two hours' heating. None of the three substances is soluble in cold conc. hydrochloric acid, and the latter contains no reducing carbohydrates after 2 hours' contact with the products, and shows but a slight reduction after two days' standing.

\section{Summary.}

It has been shown that carbohydrates of all types are readily benzylated, and various benzyl ethers obtained are described. The benzylation reaction should prove useful in the further study of carbohydrates, just as alkylation by means of methyl sulfate has proven so preëminently fruitful in this field. Moreover, some of the benzyl ethers of the carbohydrates may prove, it is hoped, technically useful products because of their properties as colloid and plastic substances, particularly since benzyl chloride is relatively inexpensive and the group increases considerably the molecular weights of the carbohydrates to which it adds.

ANn ARBor, Mrch.

[CONTRIBUtion from the Research Department of the BarrettT Company.] THE QUANTITATIVE DETERMINATION OF PHENANTHRENE. ${ }^{1}$

\section{By Arthur G. Williams.* \\ Received May 16, 1921.}

The hydrocarbon phenanthrene has been the object of much research. It occurs in relative abundance in coal tar along with carbazole and anthracene, being isomeric with the latter. Much work has naturally been directed toward using it in making dyes and, indeed, many patents have been granted for dyes of which it forms a basis. Notable among such dyes is flavinduline. Further, the morphine alkaloids contain a phenanthrene nucleus, and this fact has been most potent in giving impulse to investiga-

${ }^{1}$ Read before the Dye Division of the American Chemical Society at the Rochester Meeting, April, 1921.

* Died September 1, 1921. 